\title{
Sub-Millimeter-Wave Transmission of Silk Fabric
}

\author{
Min Zhang1, Bin Du', Wanjun Zheng' ${ }^{2}$ Xuesen Zhong1, Hong Su1, Ling Li1, \\ Chengxiang Liu ${ }^{1}$, Huawei Liang ${ }^{*}$
}

\begin{abstract}
${ }^{1}$ Shenzhen Key Laboratory of Laser Engineering, Key Laboratory of Advanced Optical Precision Manufacturing Technology of Guangdong Higher Education Institutes, College of Optoelectronic Engineering, Shenzhen University, Shenzhen, China ${ }^{2}$ Guangdong Polytechnic of Science and Technology, Zhuhai, China

Email: *hwliang@szu.edu.cn
\end{abstract}

How to cite this paper: Zhang, M., Du, B., Zheng, W.J., Zhong, X.S., Su, H., Li, L., Liu, C.X. and Liang, H.W. (2018) Sub-Millimeter-Wave Transmission of Silk Fabric. Journal of Computer and Communications, 6, 124-128.

https://doi.org/10.4236/jcc.2018.61013

Received: September 26, 2017 Accepted: December 26, 2017

Published: December 29, 2017

\begin{abstract}
Millimeter wave technology is one of the technologies developed rapidly in recent years. The research was mainly to study the transmission of millimeter wave through textile material named silk fabric. We observed the phenomenon of sub-millimeter-wave transmission and reflection of the silk fabric, and concluded that with the number of silk fabric layers increasing, effect of scattering and absorption of the millimeter wave enhanced. The conclusion could be further employed in millimeter wave and $\mathrm{THz}$ imaging, dangerous goods inspection and other security application.
\end{abstract}

\section{Keywords}

Millimeter Wave, Sub-Millimeter-Wave, Silk Fabric, Microwave Applications, Measurement Techniques

\section{Introduction}

The millimeter wave is between the infrared and the microwave, its wavelength is approximately $0.03 \mathrm{~mm}$ to $1 \mathrm{~mm}$, and the corresponding frequency is about $300 \mathrm{GHz}$ to $10,000 \mathrm{GHz}$ [1] [2]. Habitually, it is known as far-infrared by optical-workers, which is the developing area of research [3]. Millimeter-wave is like a newborn, especially the developing millimeter-wave, which open the new significant research field in infrared and microwave.

Millimeter wave has three main features: short wavelength, wide Bandwidth, and the interaction between the atmosphere. As the basis of most applications, however, these features also make scope of its application is restricted. Since the early last century, millimeter wave has been considered promising [4]. With Jo- 
sephson device and SIS device exploitation, equipment made of millimeter wave technology has higher power output, high signal to noise ratio. Technique is rapidly increasing, but in many fields, like the employment early in astronomy, medicine, simple observational science, precise analysis of matter [5] [6], even observations of supergalaxy, it is not applied in important applications because the characteristics confine the performance of the applications.

There are always many researchers working on it. However, there are very few reports on the silk fabric [7] for millimeter wave transmission [8]. By studying absorption and diffraction to millimeter wave of silk fabric, we can get some characteristics of them, which can help us to do further research on $\mathrm{THz}$ imaging.

\section{Experiment Setup}

When incident light is illuminated to the textile material, it will be of the absorption, reflection and transmission [8]. Light transmission is different for different materials, each material light absorption of the divergence is also different [9]. Through researching on silk fabric light absorption under one kind of light scattering and transmission characteristics, we are able to get the properties of the silk fabric.
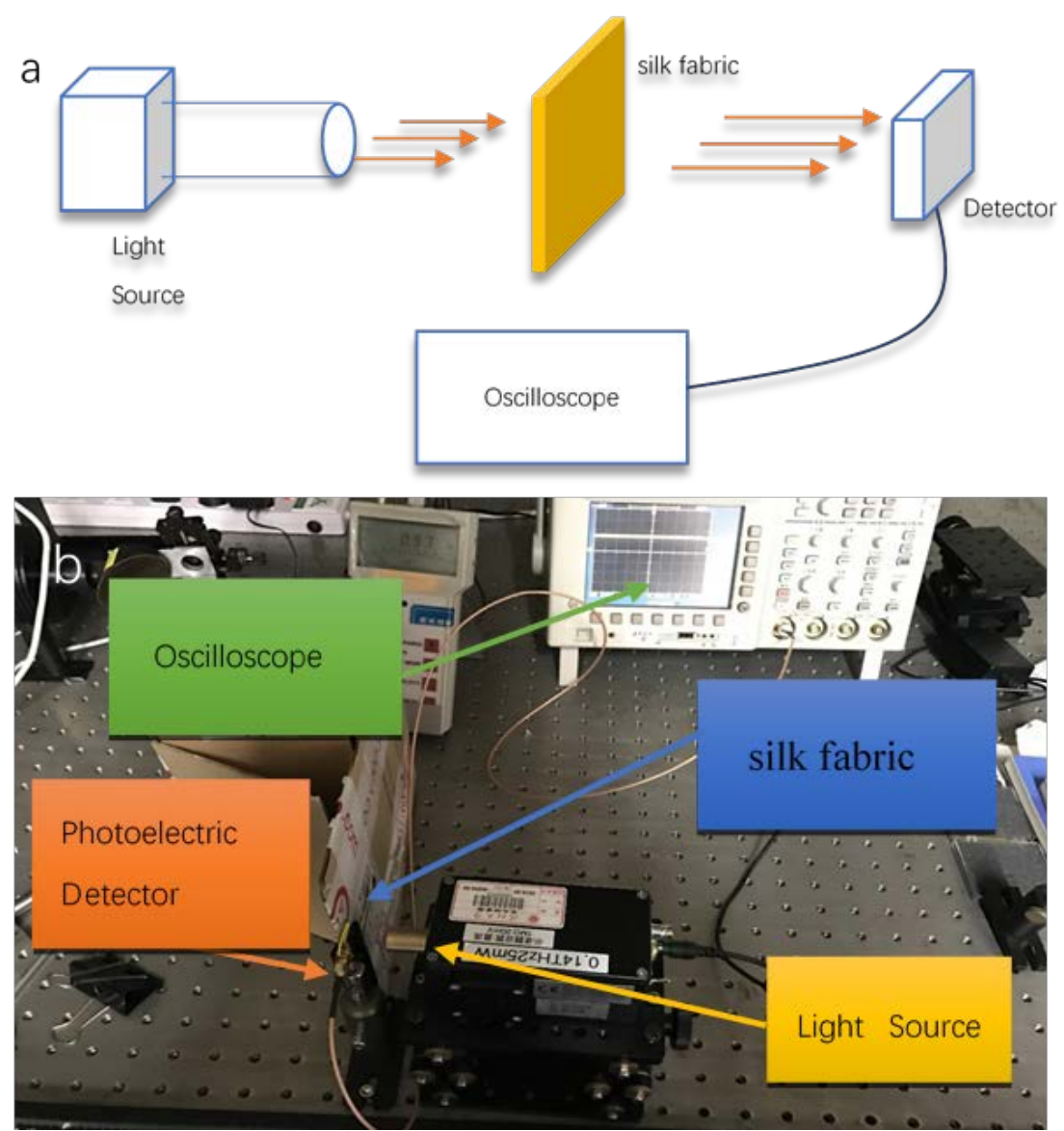

Figure 1. The experiment setup schemes. 
The experimental schematic schemes are shown in Figure 1(a), actual experimental setup diagram is shown in Figure 1(b). The light source generator emits $0.14 \mathrm{THz}$ light, then the light is illuminated on the cloth materials, some of which transmits the cloth materials (Figure 2) and is detected by the detector. At last, the oscilloscope receives the corresponding data, the screen displays it.

\section{Result and Discussion}

In Figure 3(a), we conclude that with thickness increasing, the millimeter wave transmission rate decrease. Silk fabric layer increases, the textile material hindering effect increases. Increasing of the textile material layers is equivalent to the increasing thickness of textile materials, which make increasing of the scattering and absorption degree of the textile material for millimeter wave. We get the coefficient of absorption from the transmission curve 2, which is 0.062 $\mathrm{mm}^{-1}$. In Figure 3(c) and Figure 3(d), we have used different placement to do the experiment, and have found out that the transmission is not so stable. The silk fabric is soft and it contains lots of holes which have different shape, which is easily affected by the environment, for instance, wind.

Figure 4(a) shows the data from different angle of the detector while angle between the golden mirror and beam source. We conclude that the light through the material produce the diffraction effect. Figure 4(b) shows curve that the millimeter wave transmit through the cloth material, is reflected by a golden mirror and transmit through the cloth material again, and is detected by the photoelectric detector (Figure 1(c)). The result is similar to the first experiment (Figure 1(b)). With the thickness increasing, the cloth materials absorb and scatter more millimeter wave (Figure 4(b)). The golden does absorb less light compared to the first experiment. The millimeter wave traverses through silk fabric and reflected by the golden mirror, and then is detected by the photoelectric detector.

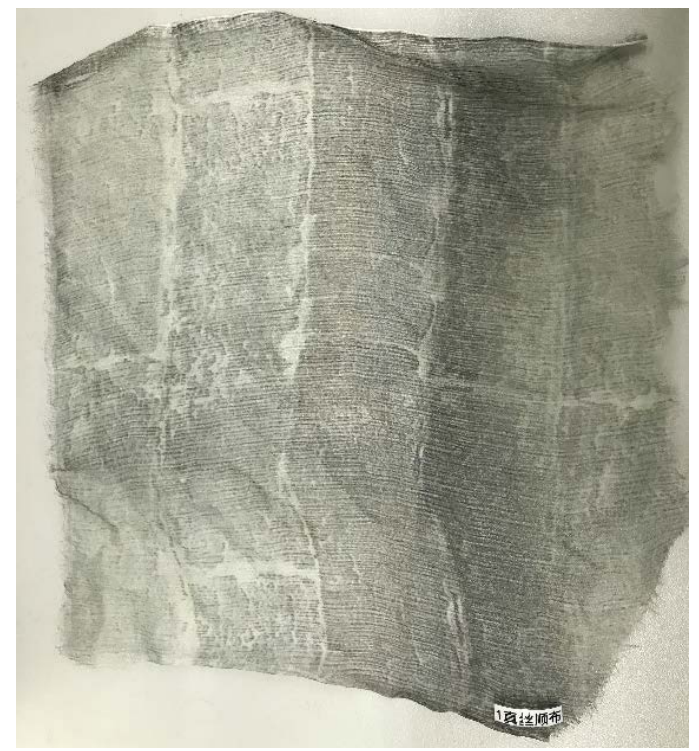

Figure 2. The fiber material used for experiment. 


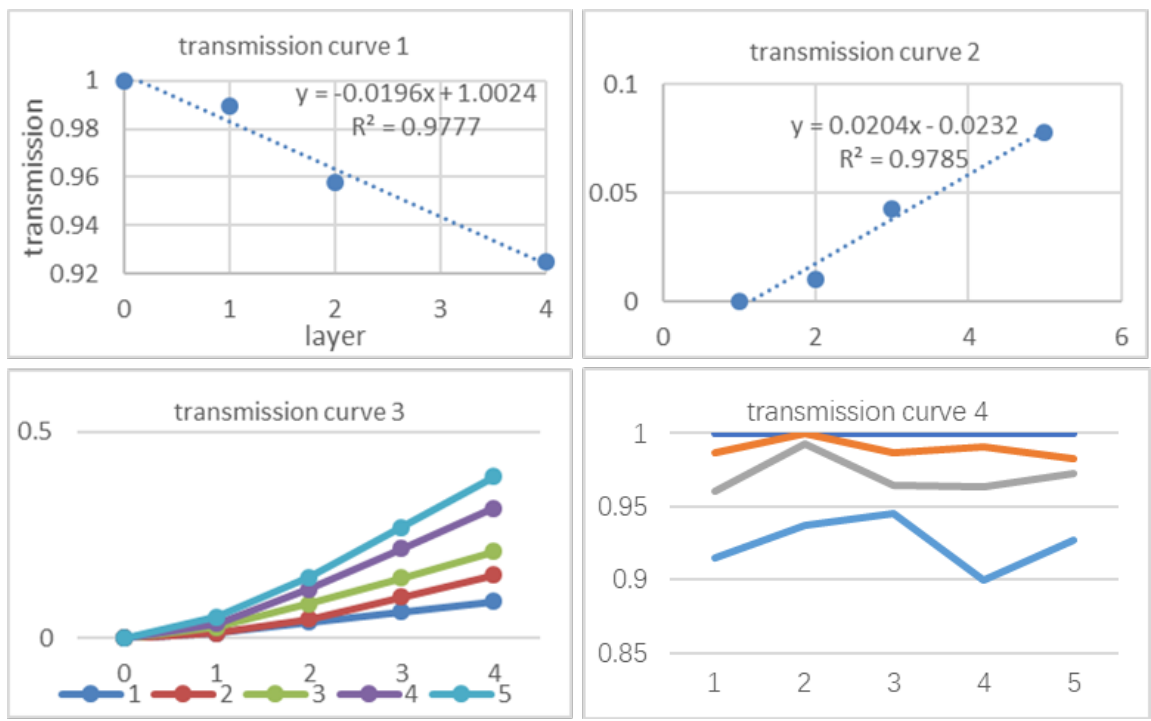

Figure 3. Millimeter-wave transmission of various layers of textile materials.
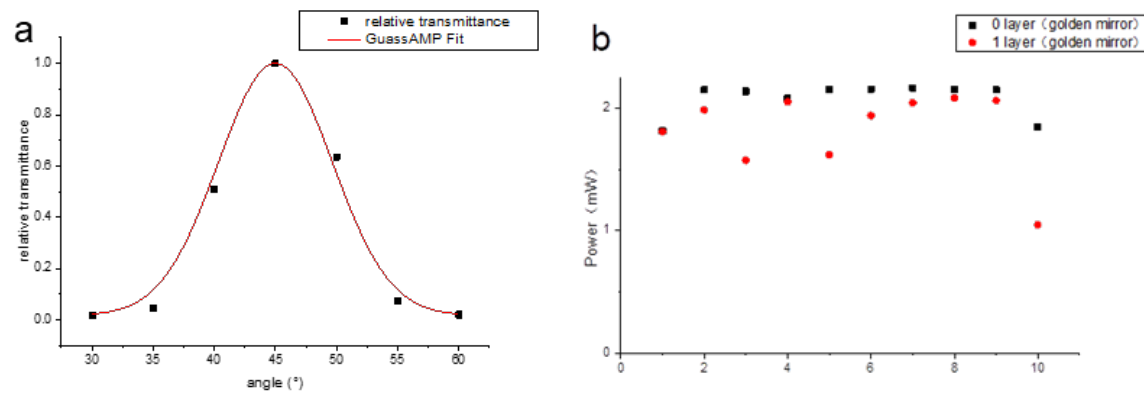

Figure 4. Different angle of the relative transmittance.

\section{Conclusion}

In summary, we have studied the phenomenon of millimeter wave through the silk fabric. The result shows that millimeter wave is easily able to traverse through the silk fabric. With the thickness increasing, the intensity of millimeter wave decreases slower and slower. We get the attenuation coefficient of the millimeter of the silk fabric. All these experiments result can be used in millimeter wave and $\mathrm{THz}$ imaging, security check in shop and subway, anthropometric shadow scanner for people to choose clothes that fit their body, and other security application.

\section{Acknowledgements}

This work was supported by the Science and Technology Innovation Commission of Shenzhen under Grants JCY20150324141711695, JSGG20150512162504354.

\section{References}

[1] Fujishima, M. (2011) BI-2-3 Millimeter-Wave/Terahertz CMOS Circuits. Proceedings of the Society Conference of IEICE.

[2] Domier, C.W., Luhmann, N.C., Park, H.K., Xia, Z. and Zhang, P. (2008) Advances 
in Millimeter Wave/THZ Plasma Diagnostics Instrumentation. Joint International Conference on Infrared and Millimeter Waves, 2007 and the 2007 International Conference on Terahertz Electronics, Irmmw-Thz, 8-11.

[3] Ihara, T. and Fujimura, K. (1996) Research and Development Trends of Millimeter-Wave Short-Range Application Systems. IEICE Transactions on Communications, 79, 1741-1753.

[4] Rebeiz, G.M. (2002) Millimeter-Wave and Terahertz Integrated Circuit Antennas. Proceedings of the IEEE, 80, 1748-1770. https://doi.org/10.1109/5.175253

[5] Du Bosq, T.W., Peale, R.E. and Boreman, G.D. (2008) Terahertz/Millimeter Wave Characterizations of Soils for Mine Detection: Transmission and Scattering. International Journal of Infrared and Millimeter Waves, 29, 769-781.

https://doi.org/10.1007/s10762-008-9376-3

[6] Bosq, T.W.D., Peale, R.E., Weeks, A., Jardine, P., Stewart, M., Grantham, J., Dillery, D., Lee, D., Muh, D. and Boreman, G. (2005) Terahertz and Millimeter Wave Transmission of Soils. Integrated Optoelectronic Devices, 2005, 1-11. https://doi.org/10.1117/12.584925

[7] Dabolina, I., Vilumsone, A. and Blums, J. (2011) Investigation of Textile Materials for Laser Light Beam Scanning. Advanced Materials Research, 222, 40-43. https://doi.org/10.4028/www.scientific.net/AMR.222.40

[8] Bjarnason, J.E., Chan, T.L.J., Lee, A.W.M. and Celis, M.A. (2004) Millimeter-Wave, Terahertz, and Mid-Infrared Transmission through Common Clothing. Applied Physics Letters, 85, 519-521. https://doi.org/10.1063/1.1771814

[9] Hemmati, H., Mather, J.C. and Eichhorn, W.L. (1985) Submillimeter and Millimeter Wave Characterization of Absorbing Materials. Applied Optics, 24, 4489. https://doi.org/10.1364/AO.24.004489 